\title{
TRIM59 facilitates the proliferation of colorectal cancer and promotes metastasis via the PI3K/AKT pathway
}

\author{
YE SUN*, BING JI*, YIFEI FENG*, YUE ZHANG, DONGJIAN JI, CHUNYAN ZHU, SEN WANG, \\ CHUAN ZHANG, DONGSHENG ZHANG and YUEMING SUN
}

Department of Colorectal Surgery, The First Affiliated Hospital of Nanjing Medical University, Nanjing, Jiangsu 210029, P.R. China

Received February 3, 2017; Accepted April 18, 2017

DOI: $10.3892 /$ or.2017.5654

\begin{abstract}
Tripartite motif-containing 59 (TRIM59) belongs to the tripartite motif (TRIM) protein family and is upregulated in various malignancies. However, its expression in colorectal cancer (CRC) is still unknown. In the present study, we examined the expression and biological function of TRIM59 in CRC. We analyzed CRC tissues and cells by quantitative real-time polymerase chain reaction. Kaplan-Meier survival analysis was used to evaluate the prognostic significance of TRIM59 in CRC patients. Furthermore, we investigated the role of TRIM59 in CRC growth and metastasis. The potential mechanism underlying the regulation of cell metastasis by TRIM59 was determined by western blotting. TRIM59 expression was conspicuously overexpressed in CRC tissues and CRC cell lines compared to that noted in the corresponding normal control cells. Patients with higher TRIM59 expression had poorer prognosis. Furthermore, knockdown of TRIM59 suppressed cell proliferation through the induction of apoptosis and inhibited migration and invasion significantly in vitro. Further investigation revealed that knockdown of TRIM59 effectively reversed the expression of epithelialmesenchymal transformation-related proteins vimentin, Snail
\end{abstract}

Correspondence to: Dr Yueming Sun, Department of Colorectal Surgery, The First Affiliated Hospital of Nanjing Medical University, 300 Guangzhou Road, Nanjing, Jiangsu 210029, P.R. China

E-mail: sunyuemingjs@163.com

*Contributed equally

Abbreviations: CRC, colorectal cancer; TRIM, tripartite motif; DMEM, Dulbecco's modified Eagle's medium; FBS, fetal bovine serum; RT-qPCR, real-time quantitative polymerase chain reaction; cDNA, complementary DNA; NC, nucleotide sequence; siRNA, small interfering RNA; TBST, Tris-buffered saline with Tween-20; EMT, epithelial-mesenchymal transition

Key words: TRIM59, colorectal cancer, EMT, PI3K/AKT pathway, biomarker and E-cadherin. Our preliminary results confirm that TRIM59 can be mediated by PI3K/AKT signaling. TRIM59 functions as an oncogene in CRC progression, which could be a novel target for the detection and treatment of CRC.

\section{Introduction}

Colorectal cancer (CRC) has the fourth-highest incidence among all cancers and the fifth-highest incidence of tumor-related mortalities across China (1). Failure of treatments to prevent and control distant metastasis is the main reason for its high mortality of $50.7 \%$ (2). Therefore, further investigation of the active proteins or pathways in CRC progression may contribute to the development of novel therapeutic targets.

The tripartite motif (TRIM) protein family contains more than 77 members that are characterized by the presence of an N-terminal region, which consists of a RING domain, 1 or 2 B-box motifs, and a coiled-coil region (3). TRIM proteins are implicated in a wide range of physiologic processes such as immunity, proliferation, antiviral process, oncogenesis and transcriptional regulation (4-8). Due to the RING domain, a large part of the TRIM protein functions as E3 ubiquitin ligases (6). Over the last decade, the role of TRIM proteins in innate immunity has been extensively studied. In addition, recent studies have shown that various members of this family, including TRIM15, TRIM25 and TRIM29, have vital roles in human tumorigenesis (9-11).

Tripartite motif-containing 59 (TRIM59), a novel TRIM family member, is related to several cancers. Zhou et al (2014) reported that TRIM59 expression is markedly increased in gastric cancer and may promote gastric carcinogenesis by promoting ubiquitination and degradation of p53 (12). Furthermore, TRIM59 can facilitate the proliferative and migratory capacity of non-small cell lung cancer (13). Mouse models of prostate cancer have shown that TRIM59 may exert its carcinogenic effects through the RB and Ras signal pathways (14). Collectively, these data suggest that TRIM59 plays a role in tumor proliferation and migration. Nevertheless, the exact role of TRIM59 in CRC is still unclear.

Therefore, the present study aimed to examine the expression and biological function of TRIM59 in CRC. 


\section{Materials and methods}

CRC patient specimens. A total of 90 human CRC tissues and their corresponding normal colorectal mucosa were surgically acquired between June 2009 and June 2011 at The First Affiliated Hospital of Nanjing Medical University (Jiangsu, China). All tissues were frozen in liquid nitrogen immediately after surgical exeresis and stored at $-80^{\circ} \mathrm{C}$. Written informed consent was obtained from all patients or their relatives. Tumor-node-metastasis (TNM) stage was determined on the basis of The National Comprehensive Cancer Network (2015.2). In the present study, patients who had accepted any neoadjuvant radiotherapy or chemotherapy were not included.

Cell culture and chemicals. Human colorectal carcinoma cell lines Caco-2, SW480, HT-29, LoVo, DLD-1, HCT116 and normal human colorectal epithelial cells (NCM460) were maintained in our laboratory and were cultured in Dulbecco's modified Eagle's medium (DMEM) supplemented with $10 \%$ fetal bovine serum (FBS) (both from Winsent Inc., St. Bruno, Quebec, Canada) $100 \mathrm{U} / \mathrm{ml}$ penicillin and $100 \mu \mathrm{g} / \mathrm{ml}$ streptomycin at $37^{\circ} \mathrm{C}$ in an incubator containing $5 \% \mathrm{CO}_{2}$. The PI3K inhibitor LY294002 was purchased from Cell Signaling Technology (Danvers, MA, USA).

RNA extraction and real-time quantitative polymerase chain reaction ( $R T-q P C R)$. RNA was extracted from tissues and cells using TRIzol reagent (Invitrogen, Carlsbad, CA, USA). Complementary DNA (cDNA) was synthesized using PrimeScript RT reagent kit (Takara, Dalian, China). RT-qPCR was conducted using a SYBR-Green PCR kit (Roche Diagnostics, Indianapolis, IN, USA) with a StepOnePlus Real-Time PCR system (Applied Biosystems, Foster City, CA, USA). The primer sequences for PCR are presented in Table I. The PCR cycling conditions were as follows: $95^{\circ} \mathrm{C}$ for $30 \mathrm{sec}, 40$ cycles of $95^{\circ} \mathrm{C}$ for $5 \mathrm{sec}$ and $60^{\circ} \mathrm{C}$ for $30 \mathrm{sec}$, and dissociation at $95^{\circ} \mathrm{C}$ for $15 \mathrm{sec}, 60^{\circ} \mathrm{C}$ for $1 \mathrm{~min}$ and $95^{\circ} \mathrm{C}$ for $15 \mathrm{sec}$. The date was analyzed using the $2^{-\Delta \Delta \mathrm{Ct}}$ method. All qRT-PCR reactions were performed in triplicate.

Small interfering RNA (siRNA) interference and plasmid transfection. siRNA targeting human TRIM59 and a scrambled nucleotide sequence (NC) were designed and synthesized by GenePharma Corporation (Shanghai, China), and then transfected into LoVo and DLD-1 cells using Lipofectamine 3000 (Invitrogen) following the manufacturer's instructions. Assays to assess the knockdown efficiency were performed $48 \mathrm{~h}$ after transfection. The sequences for the TRIM59 siRNAs were as follows: siRNA1, 5'-CCCUGA ACAUUACAGGCAATT-3'; siRNA2, 5'-CCUAUAGAUG ACCUUCAAATT-3'; siRNA3, 5'-CCACUCAAGUGCCC UAAUUTT-3'. The NC sequences were: 5'-UUCUCCGAACG UGUCACGUTT-3' (sense) and 5'-ACGUGACACGUUCGGA GAATT-3' (antisense). Expression of the TRIM59 plasmid was purchased from GeneCopoeia, Inc., Rockville, MD, USA). The primer sequences were as follows: forward primer, 5'-CA GCCTCGGACTCTAGC-3' and reverse primer, 5'-TAATAC GACTCACTATAGGG-3'.
Table I. Primer sequences used for qRT-PCR.

\begin{tabular}{|c|c|}
\hline Primer & Sequence $\left(5^{\prime}-3^{\prime}\right)$ \\
\hline GAPDH & $\begin{array}{l}\text { F 5'-ACAGTCAGCCGCATCTTCTT-3' } \\
\text { R 5'-GACAAGCTTCCCGTTCTCAG-3' }\end{array}$ \\
\hline TRIM59 & $\begin{array}{l}\text { F 5'-CCTGTGTTTGAGATAGATTTAAGAGC-3' } \\
\text { R 5'-GCAACAAGGTGAGACCCAGT-3' }\end{array}$ \\
\hline
\end{tabular}

F, forward; R, reverse; TRIM59, tripartite motif-containing 59.

Colony-formation assay. The colony-formation assay was performed to measure cell proliferation. After transient transfection with TRIM59 siRNA3, control siRNA or untreated as a negative control, 500 cells were planked/well in 6-well plates in triplicate. The culture medium was changed to DMEM $+10 \%$ FBS every second day. When visible clones were visible, each well was washed with phosphate-buffered saline (PBS) three times, fixed with methanol for $30 \mathrm{~min}$ at room temperature, and then stained with $0.05 \%$ crystal violet for $30 \mathrm{~min}$. After washing, the colonies ( $\geq 50$ cells/colony) were counted and imaged using a digital camera.

Cell-proliferation assay. The cell viability in all samples was measured using the Cell Counting Kit-8 (CCK-8; Dojindo, Tokyo, Japan) assay according to the manufacturer's protocol. Cells $\left(2 \times 10^{3}\right)$ were seeded in $100 \mu$ l complete culture medium in 96-well plates and cultured overnight. Subsequently, $10 \mu 1$ CCK-8 was added to each well at 24, 48, 72 and $96 \mathrm{~h}$. The absorbance was measured using a microplate reader at a test wavelength of $450 \mathrm{~nm}$ and a reference wavelength of $630 \mathrm{~nm}$. Experiments were performed in triplicate.

Flow cytometric analysis. After a 48-h siRNA transfection, the cells were collected and resuspended in cold PBS, and then stained with an Annexin V-FITC Apoptosis Detection Kit I (BD Biosciences, Franklin Lakes, NJ, USA) following the manufacturer's instructions. Data were analyzed using flow cytometry (Becton-Dickinson, San Jose, CA, USA).

Western blotting. Protein lysates from cells were prepared by using a RIPA kit (Beyotime, Shanghai, China) according to the manufacturer's protocols. Equivalent amounts of protein were separated on $10 \%$ sodium dodecyl sulfate-polyacrylamide gel electrophoresis gels and subsequently transferred to polyvinylidene difluoride membranes (Millipore, Bedford, MA, USA). The membranes were blocked in 5\% non-fat milk at room temperature for 2-4 h, and then incubated with primary antibodies at $4^{\circ} \mathrm{C}$ overnight. The primary antibodies included TRIM59 (1:1,000; Santa Cruz Biotechnology, Santa Cruz, CA, USA), E-cadherin, vimentin, Snail, PI3K, p-PI3K, AKT, p-AKT $(1: 1,000)$ and glyceraldehyde 3-phosphate dehydrogenase (GAPDH) (1:10,000) (all from Cell Signaling Technology). After washing three times with Tris-buffered saline with Tween-20 (TBST), the membranes were probed with secondary peroxidase-conjugated antibodies $(1: 1,000$; Beijing Biosynthesis Biotechnology Co., Ltd., Beijing, China) at room temperature for $2 \mathrm{~h}$. After washing the membranes 
A

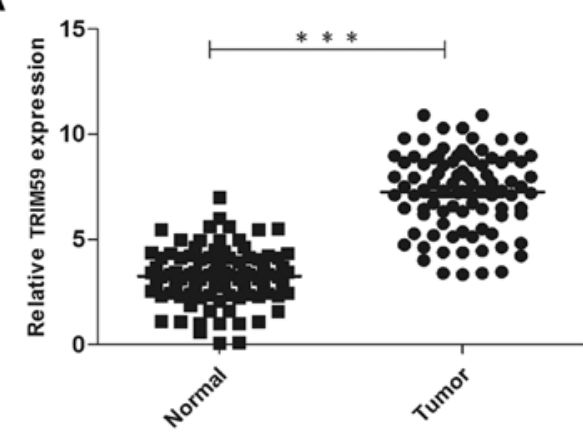

C



B

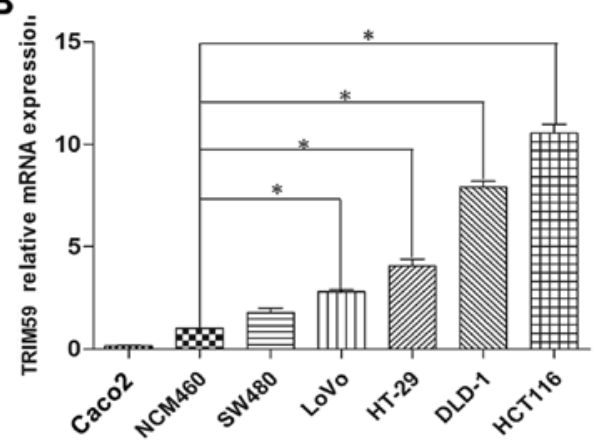

D

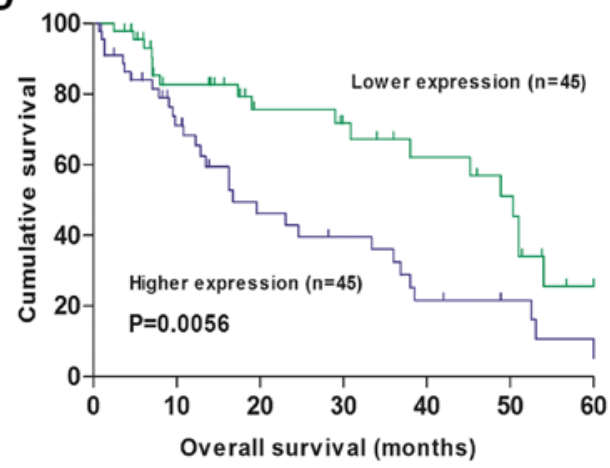

Figure 1. TRIM59 is upregulated in human CRC tissues and cell lines. (A) TRIM59 mRNA levels in CRC and normal tissues (n=90) were assessed by real-time polymerase chain reaction $\left({ }^{* * *} \mathrm{p}<0.001\right.$ compared with control non-tumorous tissues). (B) The expression level of TRIM59 was increased in four different CRC cell lines compared with normal colorectal epithelial cells (NCM460) and decreased in the Caco 2 cell line ( ${ }^{*}$ p $<0.05$ compared with NCM460 cells). (C) TRIM59 protein expression was detected by western blotting. The bar chart shows the ratio of TRIM59 protein to GAPDH. Data represent the mean \pm standard deviation of three independent experiments ( $\mathrm{p}<0.05$ compared with NCM460 cells). (D) Kaplan-Meier survival analysis of 90 CRC patients based on the TRIM59 expression level. The high-expression group showed a markedly shorter overall survival ( $\left.{ }^{*} \mathrm{p} \leq 0.05\right)$. CRC, colorectal cancer; GAPDH, glyceraldehyde 3-phosphate dehydrogenase.

three times with TBST, the bound proteins were visualized using ECL Plus (Millipore, Billerica, MA, USA) with a Bio-Imaging System. Densitometric analysis was performed to determine the relative expression of the target protein, normalized to GAPDH.

Wound-healing assay. Cells were seeded into 6-well plates at $4 \times 10^{5}$ cells/well until they reached $90-100 \%$ confluency, and a linear wound was scratched with a $200-\mu 1$ pipette tip. Thereafter, the medium was changed to serum-free DMEM. Cell migration into the artificial wound area was monitored by microscopy at two preselected time points ( 0 and $24 \mathrm{~h}$ ), and each experiment was performed in triplicate.

Transwell assay. Cell migration and invasion were assayed using a 24-well Transwell plate with polycarbonate sterile chambers ( $8 \mu \mathrm{m}$ filters; BD Biosciences) with or without Matrigel coating. Cells $\left(2 \times 10^{4}\right)$ were incubated with $100 \mu 1$ serum-free DMEM in the upper chamber and complete culture medium containing $10 \%$ FBS in the lower chamber. After incubation for $24 \mathrm{~h}$ at $37^{\circ} \mathrm{C}$, the migrated or invaded cells were stained with $0.5 \%$ crystal violet solution. Three random fields of migrated cells were selected and counted using light microscopy.
Statistical analysis. Statistical Program for Social Sciences 20.0 (SPSS, Inc., Chicago, IL, USA) and GraphPad Prism 5.0 software (GraphPad Software Inc., La Jolla, CA, USA) were used for statistical computations. Differences were analyzed using Student's t-test or one-way analysis of variance. The associations between expression levels of TRIM59 and clinicopathological features were assessed by Pearson's correlation test. Kaplan-Meier survival curves and log-rank test were carried out to analyze the survival data. Data are expressed as mean \pm standard deviation from at least three experiments. A p-value $<0.05$ was considered to indicate a statistical significance.

\section{Results}

TRIM59 expression is upregulated in CRC tissues and cells and is correlated with distant metastasis. To investigate the functional role of TRIM59 in the progression of CRC, we first determined the level of TRIM59 expression in 90 pairs of CRC tissues compared with adjacent normal tissues by using qRT-PCR. We found that TRIM59 was significantly upregulated in the tumor tissues compared with that noted in the non-cancerous tissues (Fig. 1A). We then analyzed the 
Table II. Correlation between TRIM59 expression and clinicopathological features in the colorectal cancer specimens.

\begin{tabular}{|c|c|c|c|c|}
\hline \multirow[b]{2}{*}{ Characteristics } & \multirow[b]{2}{*}{ No. } & \multicolumn{2}{|c|}{ Expression of TRIM59 } & \multirow[b]{2}{*}{ P-value } \\
\hline & & $\begin{array}{c}\text { Low } \\
\text { expression } \\
(n=45)\end{array}$ & $\begin{array}{c}\text { High } \\
\text { expression } \\
(n=45)\end{array}$ & \\
\hline \multicolumn{5}{|l|}{ Age (years) } \\
\hline$<60$ & 31 & 16 & 15 & \multirow[t]{2}{*}{0.824} \\
\hline$\geq 60$ & 59 & 29 & 30 & \\
\hline \multicolumn{5}{|l|}{ Sex } \\
\hline Male & 54 & 23 & 31 & \multirow[t]{2}{*}{0.085} \\
\hline Female & 36 & 22 & 14 & \\
\hline \multicolumn{5}{|l|}{ Tumor diameter } \\
\hline$<5 \mathrm{~cm}$ & 54 & 29 & 25 & \multirow[t]{2}{*}{0.389} \\
\hline$\geq 5 \mathrm{~cm}$ & 36 & 16 & 20 & \\
\hline \multicolumn{5}{|l|}{ TNM stage } \\
\hline $\mathrm{I} / \mathrm{II}$ & 35 & 25 & 10 & \multirow[t]{2}{*}{$0.001^{\mathrm{a}}$} \\
\hline III/IV & 55 & 20 & 35 & \\
\hline \multicolumn{5}{|l|}{$\begin{array}{l}\text { Lymph node } \\
\text { metastasis }\end{array}$} \\
\hline Positive & 42 & 27 & 15 & \multirow[t]{2}{*}{$0.011^{\mathrm{a}}$} \\
\hline Negative & 48 & 18 & 30 & \\
\hline \multicolumn{5}{|c|}{ Depth of invasion } \\
\hline $\mathrm{T} 1+\mathrm{T} 2$ & 25 & 17 & 8 & \multirow[t]{2}{*}{$0.034^{\mathrm{a}}$} \\
\hline $\mathrm{T} 3+\mathrm{T} 4$ & 65 & 28 & 37 & \\
\hline \multicolumn{5}{|c|}{ Distant metastasis } \\
\hline Positive & 14 & 3 & 11 & \multirow[t]{2}{*}{$0.005^{\mathrm{a}}$} \\
\hline Negative & 76 & 42 & 34 & \\
\hline \multicolumn{5}{|c|}{ Primary tumor site } \\
\hline Colon & 42 & 19 & 23 & \multirow[t]{2}{*}{0.398} \\
\hline Rectum & 48 & 26 & 22 & \\
\hline
\end{tabular}

Statistical analyses were performed using the Pearson's $\chi^{2}$ test. ${ }^{a} \mathrm{p}<0.05$ was considered significant. TRIM59, tripartite motif-containing 59; TNM, tumor-node-metastasis.

relationship between TRIM59 expression and the clinicopathological features of the tumor tissue samples (Table II), and classified the 90 patients into a relative high-TRIM expression group $(n=45)$ and a relative low-TRIM expression group ( $\mathrm{n}=45)$ according to the median expression of TRIM59. High TRIM59 expression levels were significantly associated with TNM staging ( $\mathrm{p}=0.001 ; \mathrm{p}<0.05)$, lymph node metastasis $(\mathrm{p}=0.011 ; \mathrm{p}<0.05)$, depth of invasion $(\mathrm{p}=0.034 ; \mathrm{p}<0.05)$, and distant metastasis $(\mathrm{p}=0.005 ; \mathrm{p}<0.05)$. Nevertheless, no significant difference was observed between TRIM59 expression and other clinicopathological characteristics. TRIM59 mRNA expression was detected in six CRC cell lines (LoVo, DLD-1, SW480, HT-29, HCT-116 and Caco2) and one normal human colon epithelial cell line (NCM460). Our results showed that the TRIM59 mRNA level was markedly higher in five CRC cell lines than that noted in the NCM460 cell line (Fig. 1B).
We also found that the protein level of TRIM59 was increased in the CRC cells (Fig. 1C). Kaplan-Meier curves showed that the overall survival for patients with high-expression of TRIM59 was significantly worse than that for such patients with low TRIM59 expression ( $\mathrm{p}=0.0056$; $\mathrm{p}<0.05)$ (Fig. 1D). Taken together, these data suggest that TRIM59 was overexpressed in CRC and may be helpful to evaluate the prognosis of CRC.

siRNA suppresses TRIM59 expression in CRC cells. To investigate the functional relevance of TRIM59 in CRC tumorigenesis, two CRC cell lines (LoVo and DLD-1) which showed high levels of TRIM59 expression and better growth behavior of all cells were selected for this assay. RT-PCR showed that TRIM59 siRNAs (siRNA1, siRNA2 and siRNA3) efficiently knocked down TRIM59 compared with cells transfected with control scrambled siRNA (NC) in the chosen cell lines (Fig. 2A), and siRNA3 showed the best inhibitory effect on TRIM59 mRNA expression. Identical results were presented by western blotting, confirming that TRIM59 protein expression was notably suppressed by siRNA3 in two cell lines (Fig. 2B). Accordingly, siRNA3 was selected for the ensuing experiments.

Depletion of TRIM59 negatively regulates proliferation of CRC cells. As TRIM59 activates proliferation in several different cell types, we examined whether it facilitates cell proliferation in human CRC. The CCK-8 assays indicated that siTRIM59-transfected CRC cells showed evidently decreased proliferation in comparison to cells transfected with NC (Fig. 3A and B). Moreover, the colony-formation assay used to further investigate the anchor-independent growth ability of transfected cells showed that TRIM59 knockdown attenuated the colony numbers (Fig. 3C and D). Collectively, these results suggested that interference of TRIM59 reduces the proliferative capacity of CRC cells.

Knockdown of TRIM59 increases apoptosis of CRC cells through the mitochondrial pathway. To investigate the effect of TRIM59 knockdown on CRC cell apoptosis, the apoptotic effect after the CRC cells were transfected with siTRIM59 was analyzed by flow cytometry. The Annexin V/PI bi-parameter method results implied that the cell apoptosis rates in the blank group, the NC group, and the siTRIM59 group were as follows: for LoVo cells: $0.39,1$ and $5.54 \%$, respectively; for DLD-1 cells: $1.16,1.70$ and $9.67 \%$, respectively. Compared with the blank group and the NC group, markedly elevated cell apoptosis rates were noted in the siTRIM59 group $(\mathrm{p}<0.05)$. However, no significant difference was observed between the blank and NC groups ( $\mathrm{p}>0.05$ ) (Fig. 4A and B). In addition, the $\mathrm{Bcl}-2$ family proteins play different roles in the regulation of apoptosis and mainly affect the mitochondrial pathway (15). In the Bcl-2 family, pro-apoptotic member Bax and antiapoptotic member Bcl-2 are active effectors and regulators, and the ratio of $\mathrm{Bcl}-2 / \mathrm{Bax}$ is usually regarded as a criterion in programmed cell death (16). RT-qPCR (Fig. 4C and D) and western blotting (Fig. 4E and F) analysis indicated that TRIM59 knockdown suppressed the expression of Bcl-2, but enhanced the expression of Bax in LoVo and DLD-1 cell lines. These results indicate that TRIM59 plays an inhibitory role in 
B

A

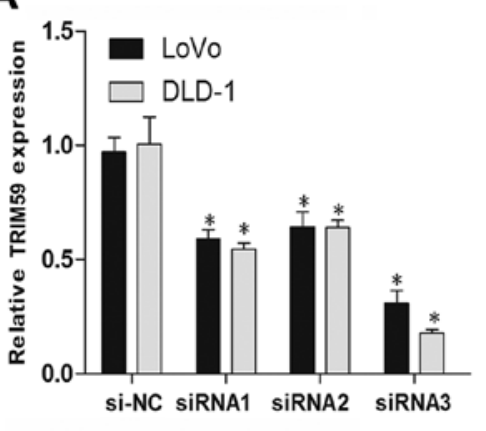



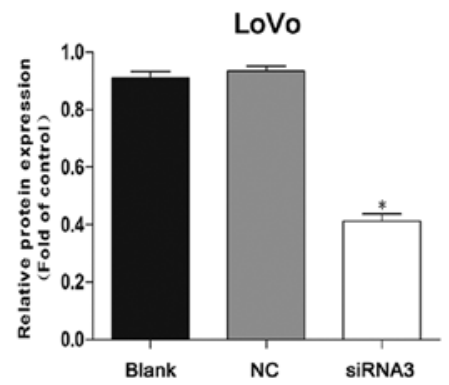

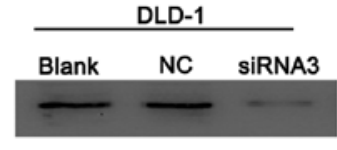

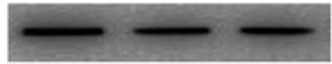

DLD-1

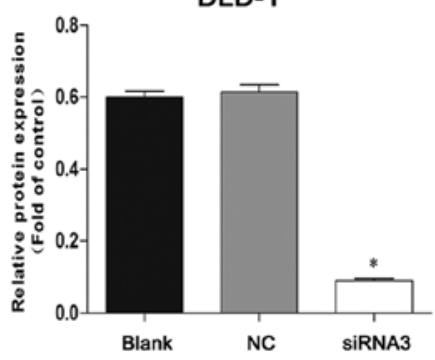

Figure 2. TRIM59 expression is suppressed by TRIM59 siRNA transfection. (A) The levels of TRIM59 mRNA were verified by RT-PCR in LoVo and DLD-1 cells after the treatment with siRNAs against TRIM59; $\mathrm{p}<0.05$ compared with NC. (B) TRIM59 protein levels were measured by western blotting and their quantification is illustrated in the bar chart; " $\mathrm{p}<0.05$ compared with NC.

A

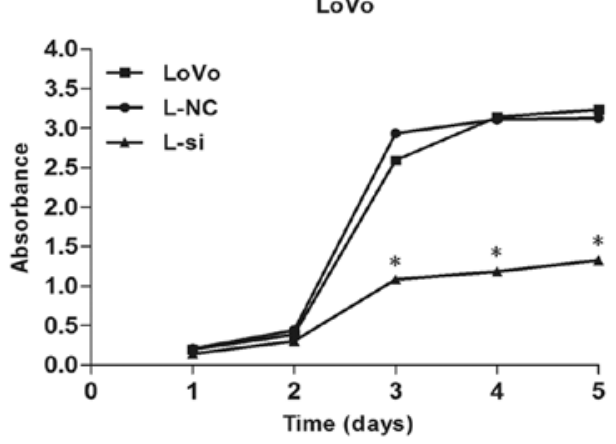

C
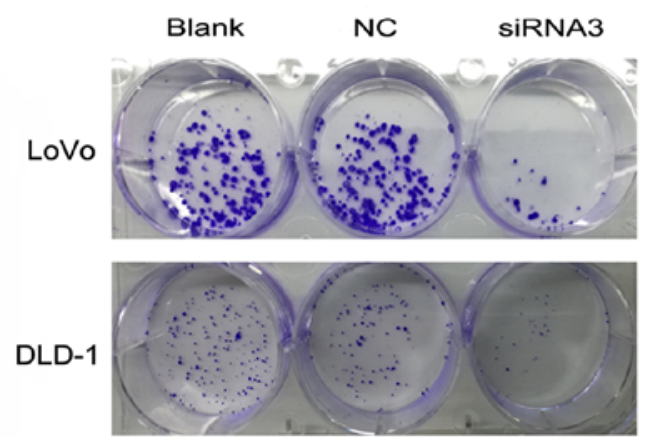

B

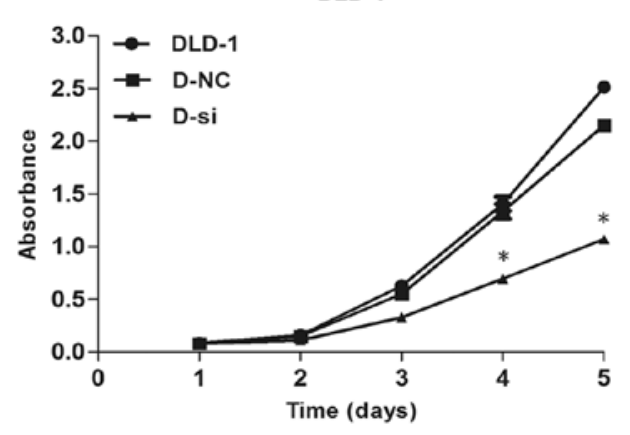

D

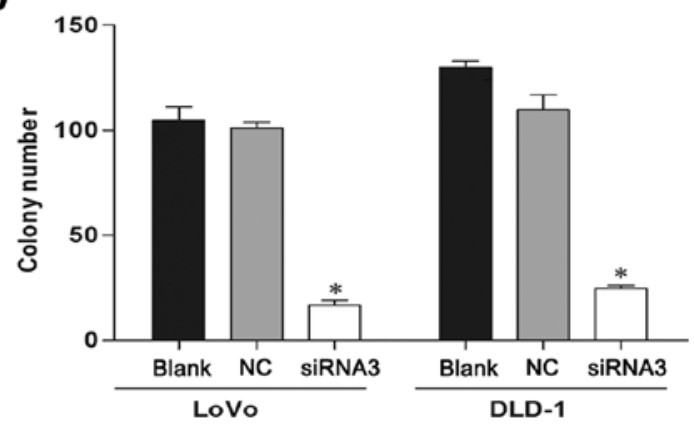

Figure 3. Effect of TRIM59 on the regulation of cell proliferation. (A and B) The CCK-8 assay showed that silencing of TRIM59 inhibited LoVo and DLD-1 cell proliferation. (C and D) The colony-forming ability of TRIM59-knockdown cells compared with NC. The colony number was normalized to cells without any treatment, which was set to $100 \%$. Data are presented as mean \pm standard deviation from three independent experiments; ${ }^{*}$ < $<0.05$.

the apoptosis of CRC cells, which proved to be linked with the balance of the $\mathrm{Bcl}-2 / \mathrm{Bax}$ ratio.

TRIM59 silencing inhibits the motility and invasiveness of CRC cells. TRIM59 has been reported to promote metastasis in gastric cancer and other cancer cells. Therefore, wound-healing and Transwell assays were performed to confirm the prometastatic properties of TRIM59 on CRC cells. The LoVo and DLD-1 cell migration effect was strikingly inhibited in the
siTRIM59 group compared with the control group (Fig. 5A). Similar results were observed from the Transwell and Matrigel assays; knockdown of TRIM59 significantly inhibited CRC cell migration and invasion (Fig. 5B). Thus, TRIM59 functioned as an oncogene accelerator by suppressing the migration and invasion of CRC cells in vitro.

TRIM59 facilitates epithelial-mesenchymal transition (EMT) in CRC cells. EMT is a critical mechanism involved 
A

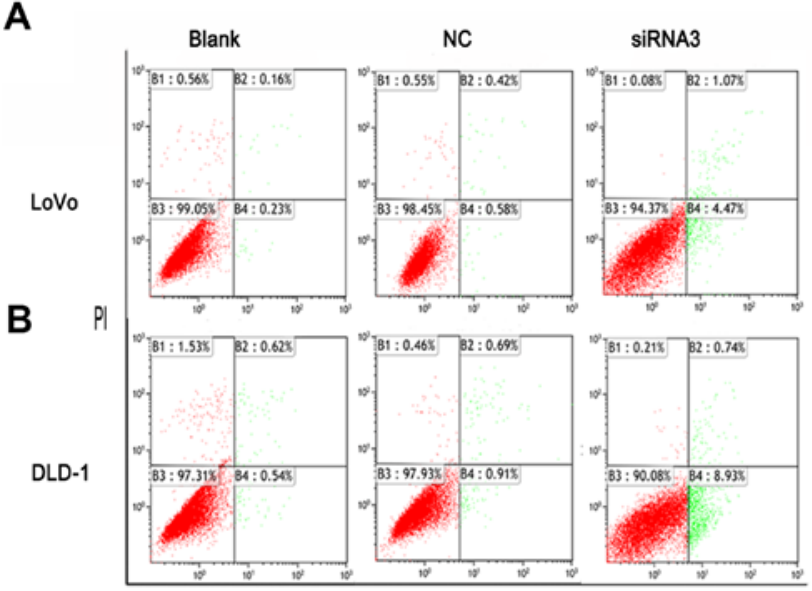

AnexinV
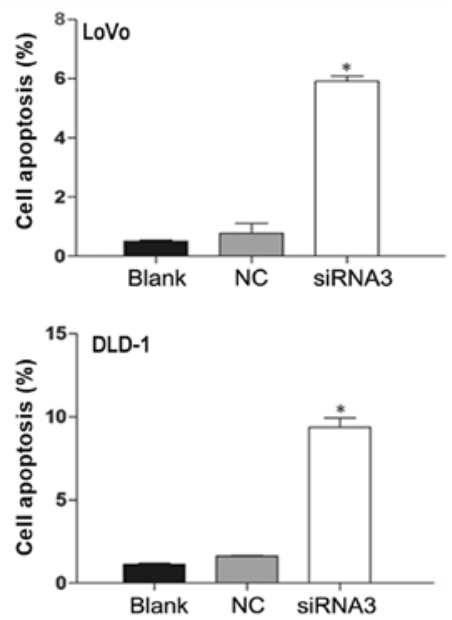

C

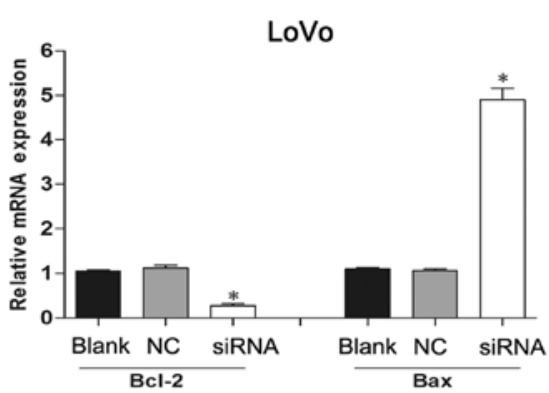

E

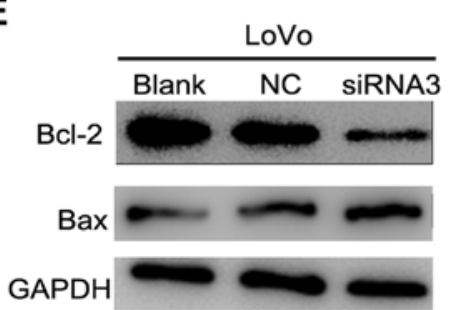

D

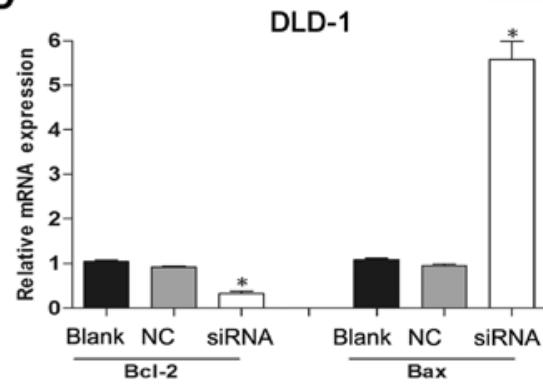

$\mathbf{F}$

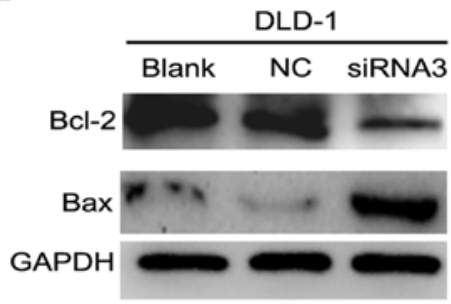

Figure 4. Knockdown of TRIM59 increases the apoptosis of CRC cells. Apoptosis of (A) LoVo and (B) DLD-1 cell lines was detected using flow cytometry; ${ }^{*} \mathrm{p}<0.05$. (C and D) mRNA expression of Bcl-2 and Bax was assessed by RT-qPCR. (E and F) Western blotting was performed to determine the protein levels of Bcl-2 and Bax. GAPDH was used as the control. GAPDH, glyceraldehyde 3-phosphate dehydrogenase.

in cell migration and invasion (17). To confirm the influence of TRIM59 knockdown on EMT in CRC cells, we evaluated the expression of EMT-associated genes in LoVo and DLD-1 cells after transfection. Western blotting and RT-PCR results showed that the epithelial marker E-cadherin was highly expressed, whereas the expression of mesenchymal marker vimentin was significantly decreased when TRIM59 was suppressed in LoVo and DLD-1 cells (Fig. 5C and D). Snail is another key regulator of EMT that functions as a transcriptional repressor of E-cadherin and other epithelial proteins $(18,19)$. In the present study, the protein levels of Snail were also markedly reduced in the TRIM59-knockdown cells. These data suggest that TRIM59 may promote the migration and invasiveness of CRC cells by regulating the EMT pathway.

Downregulation of TRIM59 restricts activation of the PI3K/AKT pathway in CRC cells. The PI3K/Akt pathway plays an essential role in cell proliferation and promotes
EMT of various types of cancer (20). Moreover, mouse prostate cancer models showed that TRIM59 may be linked with the PI3K/Akt pathway (14). Therefore, we further examined whether the PI3K/Akt pathway was involved in the TRIM59-mediated cellular response in CRC. Western blotting significantly revealed decreased levels of phosphorylated PI3K and AKT in the cells transfected with siTRIM59. However, there was no change observed in the expression of total PI3k and AKT (Fig. 6).

To investigate the role of the PI3K/Akt pathway in TRIM59-induced metastasis in CRC, a specific inhibitor of PI3K (LY294002) was used. TRIM59 was overexpressed in NCM460, which contains low expression of TRIM59, and then treated with LY294002. Transwell assays showed that overexpression of TRIM59 facilitated cell migration and invasion; however, this enhancement of metastasis was inhibited by LY294002 exposure (Fig. 7). These results confirmed the involvement of the PI3K/Akt pathway in the promotion of CRC cell migration and invasion by TRIM59. 
A
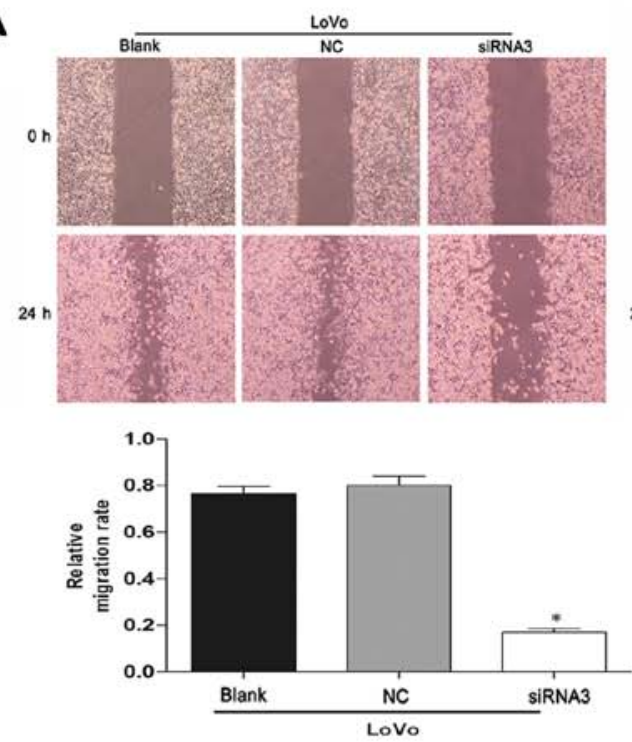

B
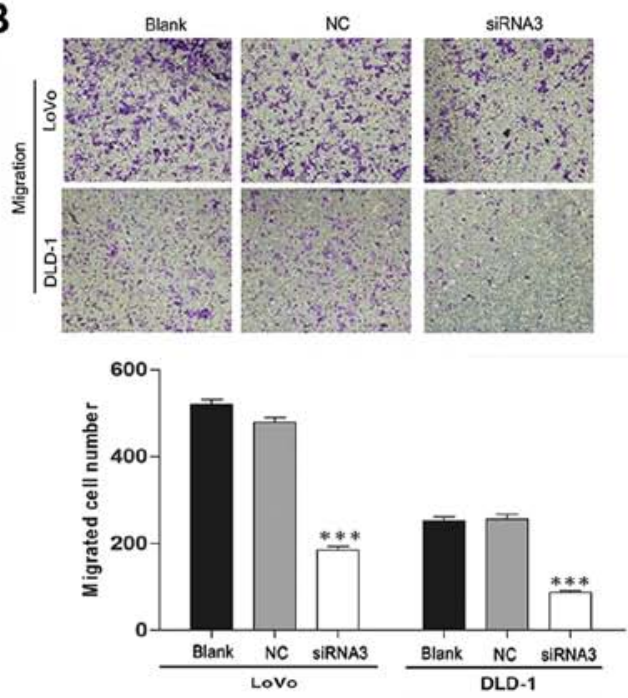

C

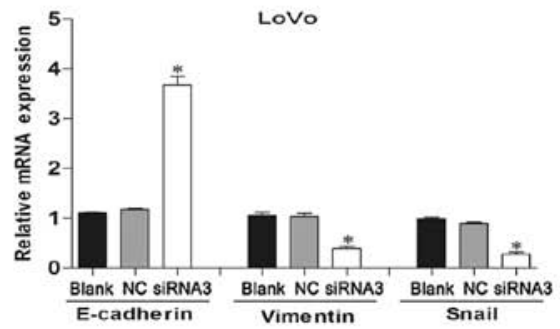

D



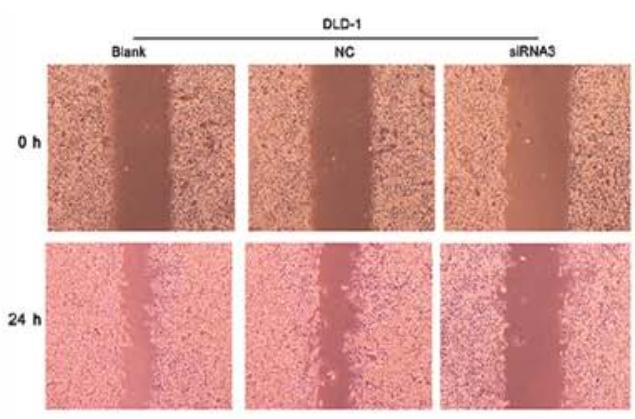
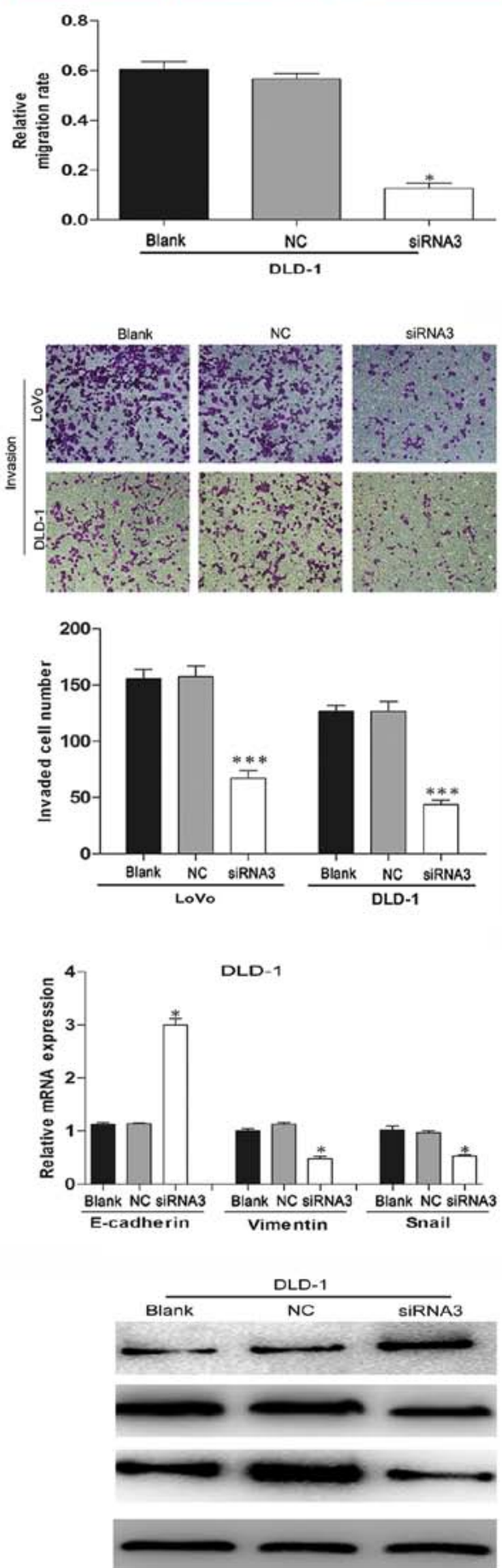

Figure 5. Knockdown of TRIM59 inhibits the migration and invasion of CRC cells. (A) Wound healing assay was performed on untransfected cells (blank), cells transfected with control siRNA (NC) and cells transfected with TRIM59 siRNA3 (siRNA3). Quantitative results are shown. Magnification, x40. (B) Migration and invasion through the Transwell assay. Numbers of migrated cells through the membrane and invaded cells through Matrigel of each cell line are shown. Magnification, $x 100$. (C and D) The effect of TRIM59 silencing on metastasis-related genes was performed by detecting mRNA and protein levels. Each assay is performed in triplicates. Data are presented as mean \pm standard deviation; ${ }^{*} \mathrm{p}<0.05$ and ${ }^{* * * *} \mathrm{p}<0.001 \mathrm{vs}$. NC. siRNA, small interfering RNA; CRC, colorectal cancer. 
A

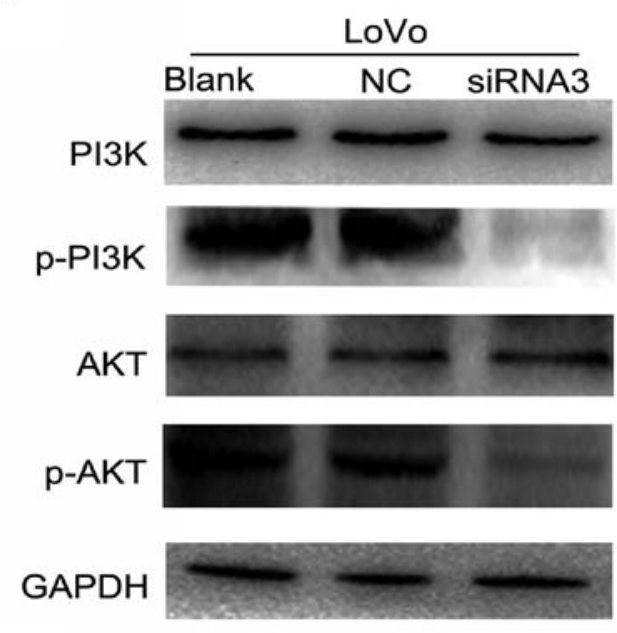

C

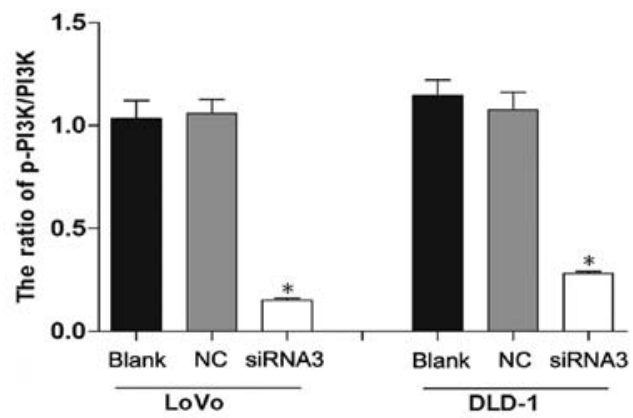

B
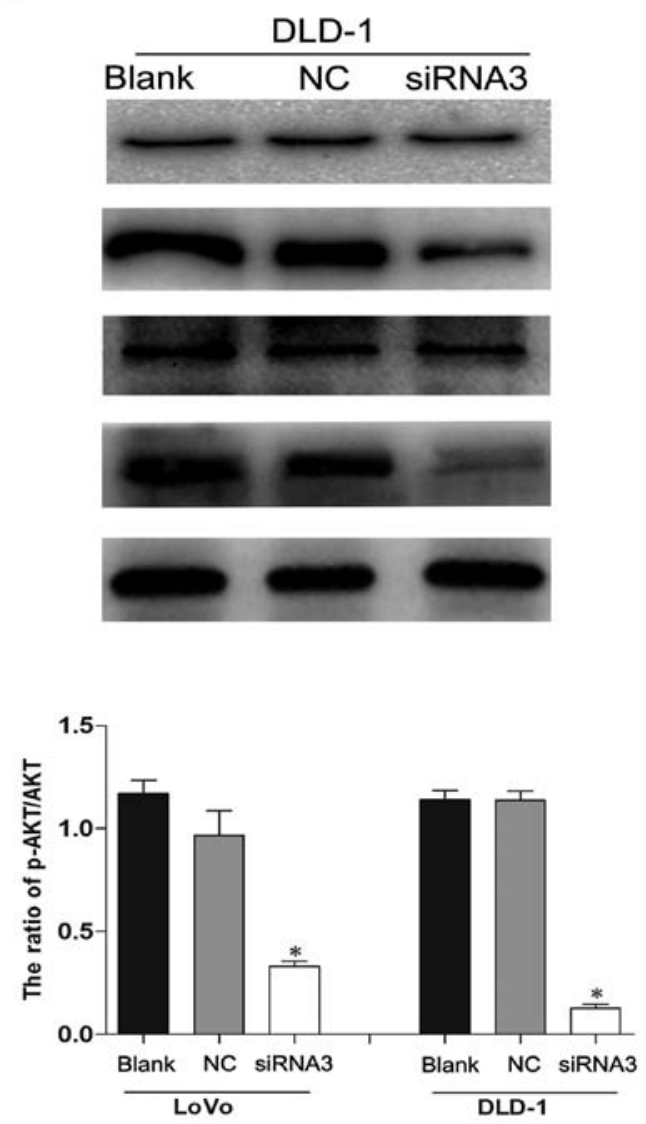

Figure 6. TRIM59 knockdown suppresses the activation of the PI3K/AKT pathways in CRC cells. Expression of PI3K, p-PI3K, AKT, p-AKT was evaluated by western blotting in (A) LoVo and (B) DLD-1 cells. (C) The relative protein expression levels of p-PI3K and p-AKT were quantified in the two cell lines. Each assay is performed in triplicate. Data are presented as mean \pm standard deviation; ${ }^{*} \mathrm{p}<0.05$ vs. NC.

\section{Discussion}

Recently, TRIM proteins have been identified as important regulators of cellular processes in a variety of human cancers (21), and various TRIM proteins have been found to be highly expressed in CRC $(9,22)$. Although the oncogenic effect of TRIM59 on progression has been confirmed in various cancers, including gastric cancer (12), osteosarcoma (23), lung (13) and cervical cancer (24), the expression and function of TRIM59 in CRC remain unclear.

In the present study, we detected the expression level of TRIM59 in CRC to assess its potential role as a clinically relevant prognostic or predictive marker. We first detected whether TRIM59 was expressed in CRC tissues and cell lines. RT-qPCR analysis of samples from 90 patients revealed that TRIM59 was overexpressed in CRC tissues as compared to normal colorectal tissues. Further analysis demonstrated that TRIM59 expression was significantly associated with TNM stage, status of lymphatic node, and presence of distant metastasis. Kaplan-Meier and Cox regression analysis revealed that the TRIM59 expression pattern was associated with poor prognosis of patients with CRC. These data indicate that TRIM59 expression could be a potential therapeutic target for diagnosis and prognosis.

We also evaluated the functions of TRIM59 in CRC cell lines. Knockdown of TRIM59 significantly suppressed cell growth in the LoVo and DLD-1 cell lines and promoted CRC cell apoptosis and decreased the ratio of Bcl-2/Bax. However, no significant changes were observed in the distribution of CRC cells in the G0/G1, S and G2/M phases, indicating that TRIM59 was not involved in cell cycle control. These data suggest that TRIM59 could suppress proliferation of CRC cells by inducing cell apoptosis.

Our clinical analysis showed that high expression of TRIM59 was associated with distant metastasis and TNM stage; we further found that TRIM59 enhanced the infiltrative capability of the CRC cells. Results of in vitro experiments indicated that silencing of the TRIM59 gene inhibited the migration and invasion of CRC cells. Metastasis of CRC remains the leading cause of the low 5-year overall survival rate in CRC (25). EMT has been reported to play critical and intricate roles in promoting tumor metastasis. This process is defined as the loss of cell-cell adhesion molecules, downregulation of epithelial differentiation markers, and transcriptional induction of mesenchymal markers (26). Thus, we determined the potential target protein levels associated with EMT-induced markers after the knockdown of TRIM59. Our results showed that depletion of TRIM59 significantly weakened vimentin and Snail expression and promoted E-cadherin expression. These data support the hypothesis that downregulation of TRIM59 may inhibit the progression of EMT by modulating EMT-related gene expression. 
A



C
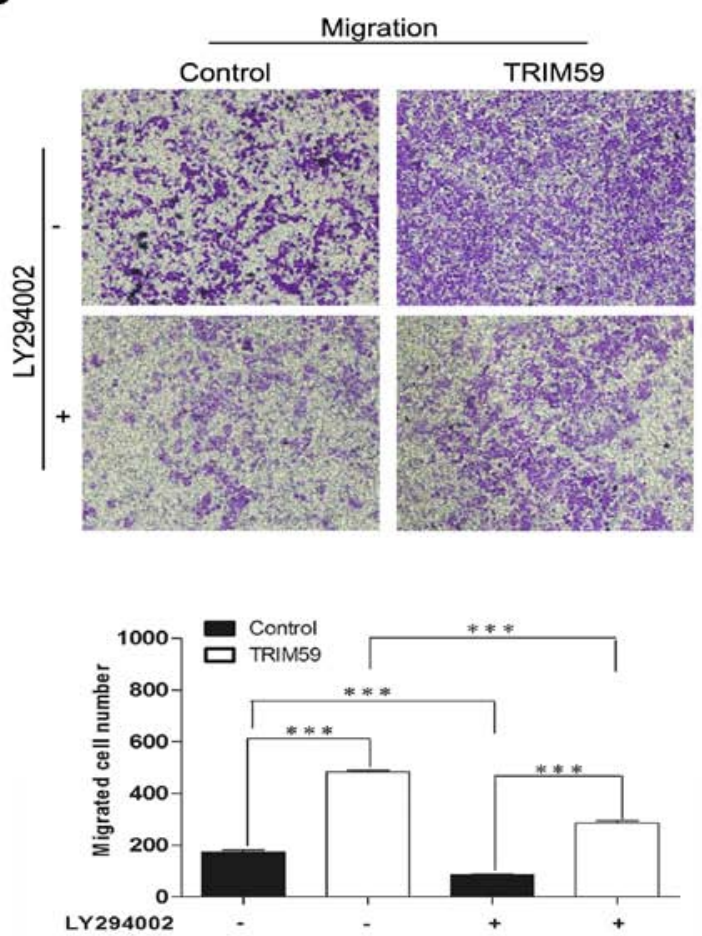

B
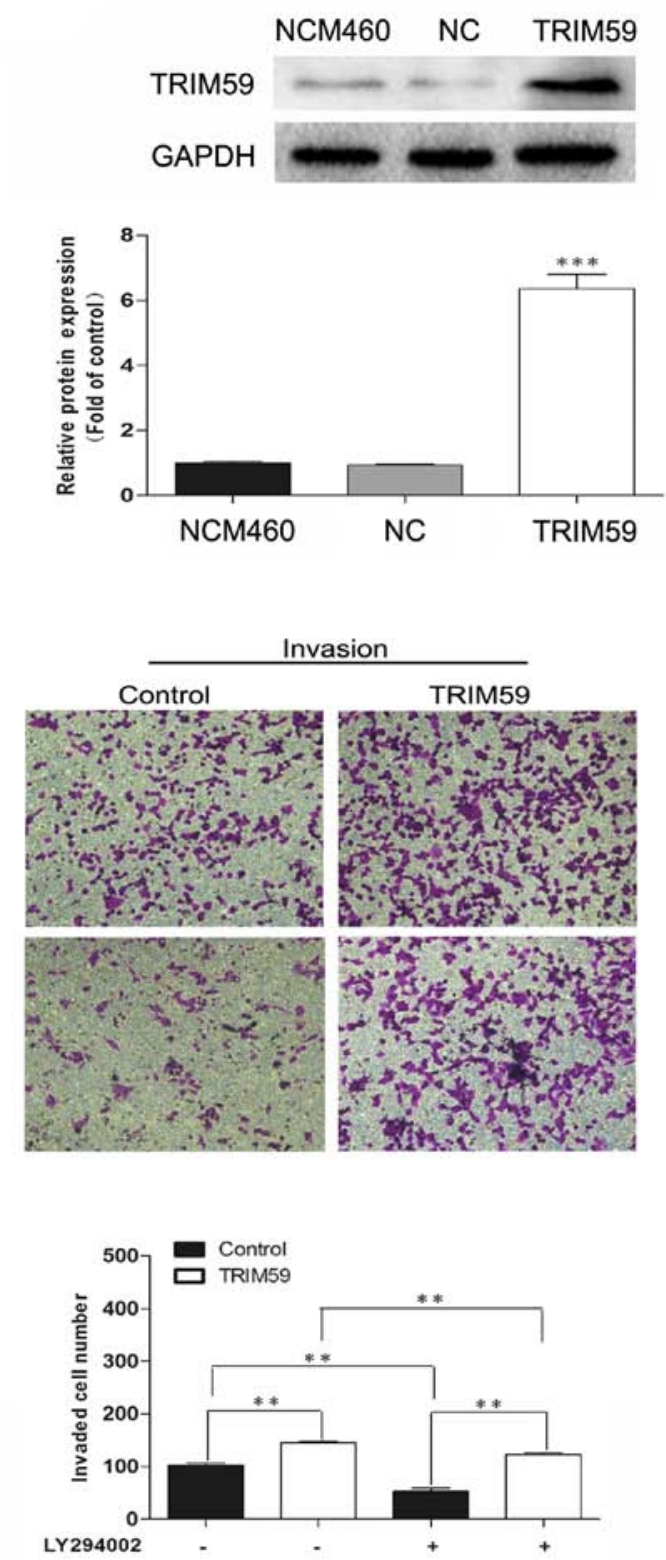

Figure 7. Overexpression of TRIM59 reduces the effects of the AKT inhibitor on the migration and invasion of CRC cells. (A) The levels of TRIM59 mRNA were verified by RT-PCR in the NCM460 cell line after treatment with the TRIM59 plasmid. (B) TRIM59 protein levels were determined using western blotting and quantification is illustrated by a bar chart. (C) Cells were transfected with control plasmid (vehicle) or TRIM59 plasmid and treated with LY294002 $(20 \mu \mathrm{M})$ for $24 \mathrm{~h}$, following which, migration and invasion assays were performed in these cells. Dimethyl sulfoxide was used as a negative control; ${ }^{* *} \mathrm{p}<0.01,{ }^{* * * *} \mathrm{p}<0.001$.

EMT is mediated by several different signaling pathways (27). Valiyeva et al reported TRIM59 as an early signal transducer in two oncogene routes: the Ras/ERK/PI3K/AKT pathway and the SV40 Tag/p53/pRB route (14). We hypothesized that oncogenic TRIM59 functions through the PI3k/AKT pathway in CRC. In the present study, we found that suppression of TRIM59 expression notably decreased the protein levels of phosphorylated PI3K and AKT, while their total protein levels remained unchanged. After treatment with LY294002, a specific inhibitor of PI3K, the facilitating roles of TRIM59 overexpression on cell migration and invasion were evidently decreased. These data suggest that TRIM59 may play an important role in CRC cell metastasis via activation of the PI3k/Akt signaling pathway. Further in-depth clinical research should focus on clarifying the role of TRIM59 in CRC.

In summary, we reported that TRIM59 expression is strikingly upregulated in human CRC, indicating that TRIM59 plays a key oncogenic role as a negative prognostic factor in patients with CRC. Moreover, TRIM59 facilitates the proliferation, migration and invasion of CRC cells and regulates cell metastasis through the PI3K/AKT pathway. Therefore, TRIM59 may serve as a potential prognostic and therapeutic target of CRC. Future studies should further investigate the mechanism by which TRIM59 is involved in the development of CRC. 


\section{References}

1. Chen W, Zheng R, Baade PD, Zhang S, Zeng H, Bray F, Jemal A, Yu XQ and He J: Cancer statistics in China, 2015. CA Cancer J Clin 66: 115-132, 2016.

2. Glockzin G, Schlitt HJ and Piso P: Therapeutic options for peritoneal metastasis arising from colorectal cancer. World J Gastrointest Pharmacol Ther 7: 343-352, 2016.

3. Yamada Y, Takayama KI, Fujimura T, Ashikari D, Obinata D, Takahashi S, Ikeda K, Kakutani S, Urano T, Fukuhara H, et al: A novel prognostic factor TRIM44 promotes cell proliferation and migration, and inhibits apoptosis in testicular germ cell tumor. Cancer Sci 108: 32-41, 2017.

4. Ozato K, Shin DM, Chang TH and Morse HC III: TRIM family proteins and their emerging roles in innate immunity. Nat Rev Immunol 8: 849-860, 2008.

5. James LC, Keeble AH, Khan Z, Rhodes DA and Trowsdale J: Structural basis for PRYSPRY-mediated tripartite motif (TRIM) protein function. Proc Natl Acad Sci USA 104: 6200-6205, 2007.

6. Gack MU, Shin YC, Joo CH, Urano T, Liang C, Sun L, Takeuchi O, Akira S, Chen Z, Inoue S, et al: TRIM25 RING-finger E3 ubiquitin ligase is essential for RIG-I-mediated antiviral activity Nature 446: 916-920, 2007

7. Khatamianfar V, Valiyeva F, Rennie PS, Lu WY, Yang BB, Bauman GS, Moussa M and Xuan JW: TRIM59, a novel multiple cancer biomarker for immunohistochemical detection of tumorigenesis. BMJ Open 2: 2, 2012.

8. Nisole S, Stoye JP and Saïb A: TRIM family proteins: Retroviral restriction and antiviral defence. Nat Rev Microbiol 3: 799-808, 2005.

9. Lee OH, Lee J, Lee KH, Woo YM, Kang JH, Yoon HG, Bae SK, Songyang Z, Oh SH and Choi Y: Role of the focal adhesion protein TRIM15 in colon cancer development. Biochim Biophys Acta 1853: 409-421, 2015.

10. Zhu Z, Wang Y, Zhang C, Yu S, Zhu Q, Hou K and Yan B: TRIM25 blockade by RNA interference inhibited migration and invasion of gastric cancer cells through TGF- $\beta$ signaling. Sci Rep 6: 19070, 2016.

11. Tan ST, Liu SY and Wu B: TRIM29 overexpression promotes proliferation and survival of bladder cancer cells through NF- $\kappa \mathrm{B}$ signaling. Cancer Res Treat 48: 1302-1312, 2016.

12. Zhou Z, Ji Z, Wang Y, Li J, Cao H, Zhu HH and Gao WQ: TRIM59 is up-regulated in gastric tumors, promoting ubiquitination and degradation of p53. Gastroenterology 147: 1043-1054, 2014

13. Zhan W, Han T, Zhang C, Xie C, Gan M, Deng K, Fu M and Wang JB: TRIM59 promotes the proliferation and migration of non-small cell lung cancer cells by upregulating cell cycle related proteins. PLoS One 10: e0142596, 2015

14. Valiyeva F, Jiang F, Elmaadawi A, Moussa M, Yee SP, Raptis L, Izawa JI, Yang BB, Greenberg NM, Wang F, et al: Characterization of the oncogenic activity of the novel TRIM59 gene in mouse cancer models. Mol Cancer Ther 10: 1229-1240, 2011.
15. Ma L and Li W: Emodin inhibits LOVO colorectal cancer cell proliferation via the regulation of the $\mathrm{Bcl}-2 / \mathrm{Bax}$ ratio and cytochrome $c$. Exp Ther Med 8: 1225-1228, 2014.

16. Zhu W, Ye L, Zhang J, Yu P, Wang H, Ye Z and Tian J: PFK15, a small molecule inhibitor of PFKFB3, induces cell cycle arrest, apoptosis and inhibits invasion in gastric cancer. PLoS One 11: e0163768, 2016.

17. Ye X and Weinberg RA: Epithelial-mesenchymal plasticity: A central regulator of cancer progression. Trends Cell Biol 25: 675-686, 2015 .

18. Kim JH, Park S, Chung $\mathrm{H}$ and Oh S: Wnt5a attenuates the pathogenic effects of the Wnt/ $\beta$-catenin pathway in human retinal pigment epithelial cells via down-regulating $\beta$-catenin and Snail. BMB Rep 48: 525-530, 2015.

19. Yook JI, Li XY, Ota I, Fearon ER and Weiss SJ: Wnt-dependent regulation of the E-cadherin repressor snail. J Biol Chem 280: 11740-11748, 2005

20. Vivanco I and Sawyers CL: The phosphatidylinositol 3-Kinase AKT pathway in human cancer. Nat Rev Cancer 2: 489-501, 2002.

21. Chen Y, Guo Y, Yang H, Shi G, Xu G, Shi J, Yin N and Chen D: TRIM66 overexpresssion contributes to osteosarcoma carcinogenesis and indicates poor survival outcome. Oncotarget 6: 23708-23719, 2015.

22. Wang J, Zhu J, Dong M, Yu H, Dai X and Li K: Knockdown of tripartite motif containing 24 by lentivirus suppresses cell growth and induces apoptosis in human colorectal cancer cells. Oncol Res 22: 39-45, 2014.

23. Liang J, Xing D, Li Z, Shen J, Zhao H and Li S: TRIM59 is upregulated and promotes cell proliferation and migration in human osteosarcoma. Mol Med Rep 13: 5200-5206, 2016.

24. Aierken G, Seyiti A, Alifu M and Kuerban G: Knockdown of tripartrtite-59 (TRIM59) inhibits cellular proliferation and migration in human cervical cancer cells. Oncol Res 25: 381-388, 2017.

25. Townsend AR, Chong LC, Karapetis C and Price TJ: Selective internal radiation therapy for liver metastases from colorectal cancer. Cancer Treat Rev 50: 148-154, 2016.

26. Thiery JP, Acloque H, Huang RY and Nieto MA: Epithelialmesenchymal transitions in development and disease. Cell 139: 871-890, 2009.

27. Chen B, Zeng X, He Y, Wang X, Liang Z, Liu J, Zhang P, Zhu H, $\mathrm{Xu} \mathrm{N}$ and Liang S: STC2 promotes the epithelial-mesenchymal transition of colorectal cancer cells through AKT-ERK signaling pathways. Oncotarget 7: 71400-71416, 2016. 